Neurosurg Focus 20 (4):E23, 2006

\title{
Efficacy of antiangiogenic targeted toxins against glioblastoma multiforme
}

\author{
Walter A. Hall, M.D., and Daniel A. Vallera, Ph.D. \\ Departments of Neurosurgery, Radiation Oncology, and Radiology, University of Minnesota Medical \\ School and the University of Minnesota Cancer Center, Minneapolis, Minnesota
}

\begin{abstract}
Object. Because the prognosis for patients with glioblastoma multiforme (GBM) remains poor, investigators have focused on developing new and more effective treatment modalities. Targeted toxins represent a new class of compounds composed of a potent protein toxin and a carrier ligand that will recognize cell surface antigens located on target tissue. A recombinant fusion protein was created that contains the translocation and catalytic portions of diphtheria toxin that are responsible for cell entry and killing, respectively, fused to the noninternalizing aminoterminal fragment portion of human plasminogen activator. This diptheria toxin-uPA fusion protein (DTAT) has the advantage over other fusion proteins of targeting malignant glioma cells and the endothelial cells of the neovasculature that express the urokinase-type plasminogen activator receptor (uPAR). Another protein, DTAT13, was synthesized to target UPAR on the neovasculature and the UPAR and interleukin-13 receptor-expressing GBM cells. The authors describe the in vitro and in vivo efficacy of DTAT and DTAT13 against GBM.

Methods. The in vitro cytotoxicity of DTAT and DTAT13 was measured using cell proliferation assays. In vivo studies were performed in which DTAT, DTAT13, or a control protein was injected directly into GBM flank tumors in athymic nude mice. Tumor volume was assessed over time and analyzed using the Student t-test. The systemic organ effects of DTAT and DTAT13 were examined functionally and histologically in tumor-free C57BL/6 mice.

In vitro, DTAT and DTAT13 were found to be highly potent and selective against U118MG, U87MG, and U373MG GBM cell lines and human umbilical vein endothelial cells. In vivo, DTAT and DTAT13 both caused a statistically significant $(\mathrm{p}<0.05)$ regression of U87MG GBM flank tumors when administered every other day at $10 \mu \mathrm{g} / \mathrm{day}$ for five doses. No tumor regression was seen in control animals. Both DTAT and DTAT13 had little effect on histological findings in the liver, kidney, spleen, and lungs. Serum analysis did not demonstrate an effect on blood urea nitrogen levels, but liver alanine aminotransferase levels rose to statistically significant $(\mathrm{p}=0.046)$ but not life-threatening levels. Also, DTAT13 was less toxic than DTAT in studies of mortality rates.

Conclusions. Both DTAT and DTAT13 might have potential for clinical application against GBM because of their ability to target both the tumor cells and neovasculature simultaneously with an absence of serious systemic side effects. The discovery that DTAT13 was less toxic than DTAT indicated that the bispecific fusion protein might target a broader subset of antigenetically diverse patients with tumors while reducing the systemic exposure to toxin that would be necessary if two agents were administered separately.
\end{abstract}

KEY WORDS $\bullet$ brain neoplasm $\bullet$ diphtheria toxin $\bullet$ glioblastoma multiforme
immunotoxin $\bullet$ interleukin-13 $\bullet$ urokinase $\bullet$ plasminogen activator

$\mathrm{C}$ URRENT treatments for GBM that include surgery, radiation therapy, and chemotherapy are inadequate to sustain a durable response. The estimated 2-year survival for patients is approximately $20 \%{ }^{2,15}$ Previous therapeutic approaches were hampered by the inability to identify an appropriate tumor-specific marker that could be tar-

Abbreviations used in this paper: ALT = alanine aminotransferase; ATF = aminoterminal fragment; BUN = blood urea nitrogen; $\mathrm{DT}=$ diphtheria toxin; DTAT $=$ diptheria toxin-uPA fusion protein; $\mathrm{EGF}=$ epidermal growth factor; GBM = glioblastoma multiforme; HUVEC = human umbilical vein endothelial cell; $\mathrm{IC}_{50}=$ median inhibitory concentration; $\mathrm{IgG}=$ immunoglobulin $\mathrm{G}$; IL = interleukin; PBS = phosphate-buffered saline; uPA = urokinase-type plasminogen activator; $\mathrm{uPAR}=\mathrm{uPA}$ receptor; $\mathrm{VEGF}=$ vascular endothelial growth factor. geted to GBM. The identification of certain cell-surface receptors that are preferentially overexpressed on GBM has led to the development of fusion proteins that target this type of cancer. Fusion proteins are a new class of compounds that are primarily used against malignant brain tumors and are composed of a targeting ligand attached to a potent toxin. ${ }^{2,12}$ Some problems that have been encountered and have slowed the development of these agents include high intratumoral pressures and unfavorable vascular dynamics that prevent homogeneous drug distribution within the tumor. ${ }^{4}$ Direct intratumoral delivery of fusion proteins through a technique termed convection-enhanced delivery may circumvent issues associated with drug delivery by enabling the accumulation of high local concentrations within tumor tissue and by lowering the systemic exposure to the 
W. A. Hall, and D. A. Vallera

compound for nontargeted organs. ${ }^{3,12}$ Because of the bloodbrain barrier, which interferes with the migration of compounds into and out of the brain, the delivery of fusion proteins directly into brain tumors via convection-enhanced delivery has resulted in significant therapeutic results in early-phase clinical trials. ${ }^{2,3,6,12,15}$

Specific cell-surface antigens that have been identified by investigators as potential targets for fusion proteins on GBM are the receptors for iron-loaded transferrin, IL-13, IL-4, and EGF. Unfortunately, there is no single receptor that is expressed on all GBM, and the receptor expression from one GBM to another can vary. Although some nontarget human cells can express these receptors, their expressions are limited, which creates a safe therapeutic window that allows for receptor-mediated cytotoxicity in tumors. Because of the inconsistent expression of cell-surface receptors in human GBM, we sought to develop a targeted toxin that would recognize uPAR that is expressed on both GBM cells and the neovasculature that provides nutrients to the tumor. There is extensive literature summarizing the expression of uPAR on GBM and endothelial cells. ${ }^{5,8}$ The ability to target the neovasculature could prove to be of significant clinical benefit because investigators have shown that the destruction of the tumor microvasculature by fusion proteins inhibits tumor growth. ${ }^{11}$ The potent bacterial toxins that have been coupled to the carrier ligands have been either mutated pseudomonas exotoxin or mutated DT.

The compound that we constructed, DTAT, is a hybrid molecule that uses the ATF of uPA that includes the molecular binding region. ${ }^{15}$ The ATF domain lacks the catalytic domain of uPA but possesses an EGF-like or growth factor domain that comprises the receptor binding sequence of human uPA. ${ }^{1}$ The catalytic region of DT was attached upstream of the ATF to render the molecule cytotoxic, a condition in which a single molecule of DT can kill a cell. ${ }^{2,12,15}$ To enhance the cytotoxicity of DTAT, the translocation-enhancing region was included in its design. ${ }^{15}$ Because receptor expression in GBM is heterogeneous, we added the ATF of uPA to the fusion protein DT-IL13, which is composed of IL-13 and a mutated DT, to create DTAT13. ${ }^{14}$ This molecular alteration was performed in such a way as to retain the site-specific binding of both ligand components to broaden the reactivity against GBM. In addition to targeting GBM, DTAT13 also targets the tumor vasculature with reduced toxicity because there is only one DT molecule for each bispecific DTAT13 molecule. The in vitro and in vivo antivascular activity and anti-GBM activity of both DTAT and DTAT13 will be described with consideration for any toxic side effects.

\section{Materials and Methods}

\section{Recombinant DTAT and DTAT13}

The technique for synthesizing recombinant DTAT has been previously described..$^{15,16}$ To construct DTAT, an NcoI/XhoI DNA fragment was cloned encoding the ATF of human uPA (Fig. 1 upper). A DNA fragment encoding truncated DT (amino acids 1-389, termed $\mathrm{DT}_{390}$, including the methionine start codon) was spliced downstream. The two genes were linked by an EASGGPE linker, which is a fragment of domain 3 of pseudomonas exotoxin that previously has been shown to be a useful intermolecular linker. Attempts at positioning DT on the $3^{\prime}$ end of the molecule or eliminating the linker resulted in reduced activity, probably due to steric hindrances of the catalytic re-
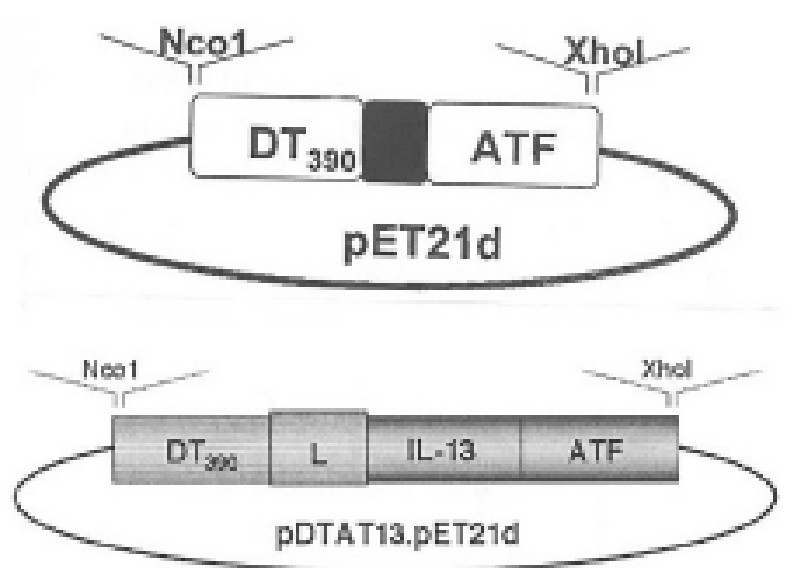

FIG. 1. Upper: Construct encoding the DTAT fragment of the $u P A$ gene fragment used in these studies. An NcoI/XhoI gene fragment was cloned by polymerase chain reaction and splice overlap extension, encoding $\mathrm{DT}_{390}$, an EASGGPE linker (black box), and the downstream 135-amino acid ATF from uPA. The gene was cloned into the pET21d expression vector, forming the plasmid pDTAT.pET21d. Lower: Construct encoding the DTAT13 gene fragment used in these studies. An $\mathrm{NcoI} / \mathrm{XhoI}$ gene fragment was cloned by polymerase chain reaction and splice overlap extension, encoding the first 389 amino acids of DT, a seven-amino acid linker EASGGPE (L1), the first 112-amino acid mature peptide of human IL-13, a 15-amino acid linker (G4-S) $)_{3}$ (L2) and the 135amino acid ATF from uPA. The gene was subcloned into the pET21d expression vector, resulting in the plasmid pDTAT13. pET21d. $\mathrm{L}=$ linker.

gion of DT or ligand binding. The DTAT hybrid gene was cloned into a vector under the control of an inducible promoter, and protein was expressed in Escherichia coli and then purified from inclusion bodies. The $\mathrm{DT}_{390}$ moiety of the protein had full enzymatic activity compared with native DT in adenosine $5^{\prime}$-diphosphate-ribosyl-transferase assays.

The DTAT13 gene consists of the first 389 amino acids of DT, an EASGGPE linker, 112 amino acids of IL-13 peptide, a 15 amino acid (G4-S) ${ }_{3}$ linker, and the 135 amino acid ATF from uPA (Fig. 1 lower). ${ }^{14}$ The DTAT13 fragment was subcloned into NcoI and XhoI cut pET21d expression vector (Invitrogen, Carlsbad, CA) to form DTAT13/pET21d. The $E$. coli cells were transformed with DTAT13/ pET21d, and protein expression was induced using isopropyl- $\beta-D-$ thiogalactoside. Then DTAT13 was isolated from inclusion bodies, solubilized, renatured, and purified using ion-exchange chromatography (Q Sepharose Fast Flow; Sigma Chemical Co., St. Louis, MO). Protein concentration was determined using Bradford assay (Coomassie Plus Protein Assay Reagent; Pierce, Rockford, IL). Protein purity was analyzed using $10 \%$ Tris- $\mathrm{HCl}$ sodium dodecyl sulfatepolyacrylamide gel electrophoresis (Bio-Rad, Richmond, CA).

\section{Cell Lines and Antibodies}

The GBM cell lines U118MG, U87MG, U373MG, and T98G were derived from human tumors and purchased from American Type Culture Collection (Rockville, MD). Daudi (a cell line derived from human Burkitt lymphoma), Neuro2a (a murine neuroblastoma cell line), and SKBR3 (a human mammary gland adenocarcinoma cell line) were used as control cell lines. They were obtained from American Type Culture Collection as well. Cell lines were maintained in RPMI 1640 medium (BioWhittaker, Walkersville, MD) supplemented with $10 \%$ heat-inactivated fetal bovine serum (BioWhittaker), $2 \mathrm{mM}$ L-glutamine (Life Technologies, Rockville, MD), $0.1 \mathrm{mM}$ nonessential amino acids, $1.0 \mathrm{mM}$ sodium pyruvate, $100 \mathrm{U} / \mathrm{ml}$ penicillin, and 100 $\mu \mathrm{g} / \mathrm{ml}$ streptomycin (Life Technologies). The HUVECs were obtained from Dr. S. Ramakrishnan (University of Minnesota) and were 
maintained in Medium 199 containing epidermal cell growth media supplement (BioWhittaker), 15\% heat-inactivated fetal bovine serum, $100 \mathrm{U} / \mathrm{ml}$ penicillin, and $100 \mu \mathrm{g} / \mathrm{ml}$ streptomycin. All the cells were maintained at $37^{\circ} \mathrm{C}$ in a humidified atmosphere of $5 \% \mathrm{CO}_{2} / 95 \%$ air and passaged two to three times per week.

Blocking experiments were performed using polyclonal rabbit anti-human urokinase IgG as well as murine IgG2a monoclonal antibody against human uPAR purchased from American Diagnostica (Greenwich, CT). ${ }^{15}$ Monoclonal antibody against human IL-13 (R\&D Systems, Minneapolis, MN) also was used for neutralization experiments. ${ }^{14}$ Anti-IL-4 antibody (rat anti-mouse IgG1 from clone 11B11) was used as a control antibody. To enhance human tumor growth in nude mice, rabbit anti-asialoGM1 (Wako Chemicals USA, Richmond, VA) was added. ${ }^{15}$

\section{Proliferation Inhibition Assay}

The in vitro cytotoxicity of DTAT and DTAT13 was measured by the amount of inhibition of DNA synthesis they produced. ${ }^{7}$ Cells were plated at $10^{4} /$ well in $100 \mu \mathrm{l}$ of culture medium in a 96-well flat-bottomed tissue-culture plate and incubated overnight at $37^{\circ} \mathrm{C}$ in a humidified atmosphere of $5 \% \mathrm{CO}_{2} / 95 \%$ air. Various concentrations of DTAT or DTAT13 diluted in culture medium were added in $100-\mu l$ increments and cultured for 48 or 72 hours. One $\mu \mathrm{Ci}$ of [methyl- ${ }^{-3} \mathrm{H}$ ] thymidine (Amersham Pharmacia Biotech UK, Little Chalfont, United Kingdom) was added to each well for the last 8 hours of incubation. Cells were harvested and washed on glass fiber filters, and the associated radioactivity was counted. All concentrations were tested in triplicate and expressed as a percentage of the control response according to the equation (counts per minute in targeted-toxin treated wells/counts per minute in untreated wells) $\times 100$. The concentration of DTAT and DTAT13 at which the $\mathrm{IC}_{50}$ of DNA synthesis occurred was calculated. For blocking experiments, antibodies were preincubated with the toxins for 30 minutes at $37^{\circ} \mathrm{C}$ before adding them to the cells.

\section{Mouse Tumor Experiments}

Six- to eight-week-old female athymic nu/nu nude mice (for tumor studies) and C57BL/6 mice (for toxicity experiments) were purchased from the National Institutes of Health (Bethesda, Maryland). They were maintained in microisolator cages under specific pathogen-free conditions as set forth by the Department of Research Animal Resources (University of Minnesota, Minneapolis, MN). On Days 2 and 4, $25 \mu \mathrm{l}$ of anti-asialoGM1 diluted in $175 \mu \mathrm{l}$ PBS was injected intraperitoneally into each nude mouse. Anti-asialoGM1 is an immunosuppressive agent that reacts with natural killer cells, monocytes, and fetal thymocytes in mice to facilitate tumor growth. ${ }^{17}$ On Day 0 , the human GBM cell lines U118MG, U87MG, and U373MG $\left(6 \times 10^{6}\right.$ $0.1 \mathrm{ml}$ culture medium) were injected subcutaneously into the right flanks of the mice. Each treatment group consisted of four or five animals. Tumor size was measured using calipers, and the volume was calculated by multiplying height by width by length. Treatment was initiated when the tumors reached a volume of at least $0.1 \mathrm{~cm}^{3}$. Animals bearing U118MG tumors received a $10-\mu \mathrm{g}$ intratumoral injection of DTAT diluted in $50 \mu \mathrm{l}$ of PBS every other day for five doses. Animals with U373MG tumors received a $10-\mu \mathrm{g}$ intratumoral injection of DTAT13 every other day for a total of five doses. Tumors in control mice were treated with PBS or an irrelevant fusion protein that contained human IL-2 and DT, DT-IL2 Tumor size and body weight were recorded every 2 to 3 days.

\section{Mouse Toxicity Experiments}

In studies to determine the effects of DTAT and DTAT13 on animal body weight, U87MG tumor-bearing mice received five intratumoral injections every other day of either $20 \mu \mathrm{g}$ of DTAT or $24 \mu \mathrm{g}$ of DTAT13. Control animals received PBS. Body weight was recorded every 2 to 3 days. ${ }^{14}$

In some of the toxicity studies, mice were treated with intracranial injections of DTAT or DTAT13. ${ }^{14}$ Groups of five to 10 mice were weighed and then anesthetized with $1 \mathrm{mg}$ of ketamine $\mathrm{HCl}$ and 0.1 $\mathrm{mg}$ of xylazine. Each anesthetized mouse was positioned in a Kopf stereotactic frame (Kopf Instruments, Tujunga, CA), and the skin on its head was prepared with betadine. A small incision was made down to the skull, and the fascial tissue was scraped with a wooden dowel. A $0.5-\mathrm{mm}$ bur hole was made in the skull at a location 1.5 to $2 \mathrm{~mm}$ from the right of the midline and $1 \mathrm{~mm}$ anterior to the coronal suture. A Hamilton syringe (Hamilton Co., Reno, NV) with a 30-gauge needle was mounted on the Kopf stereotactic frame, and the needle was inserted into the right hemisphere of the mouse's brain to a depth of $2.5 \mathrm{~mm}$. After 5 or $10 \mu \mathrm{l}$ of DTAT or DTAT13 was injected, the needle was slowly withdrawn to prevent any back flow of the fusion protein. The incision was closed with sutures and the animal was allowed to recover in a warmed cage.

\section{Systemic Toxicity of DTAT}

Systemic toxicity was assessed using BUN and ALT serum assays performed on Kodak Ektachem clinical chemistry slides on a Kodak Ektachem 950 clinical analyzer (Eastman Kodak, Rochester, NY) at the University of Minnesota Medical Center, Fairview (Minneapolis, $\mathrm{MN}) .{ }^{15}$ Groups of five C57BL/6 mice each received DTAT, PBS, or a control fusion protein, $\mathrm{DT}_{390}$-anti-CD3sFv, which has been shown to cause organ toxicity. ${ }^{17}$ On the day following the last injection, the mice were killed. Individual serum samples were collected by bleeding the heart and then measured for BUN and ALT levels. The minimum specimen volume was $11 \mu \mathrm{l}$ for each assay. The BUN assay was read spectrophotometrically at $670 \mathrm{~nm}$, and in the ALT assay the oxidation of NADH was used to measure ALT activity at $340 \mathrm{~nm}$.

\section{Histological Analysis of DTAT}

To determine the efficacy of DTAT, a group of C57BL/6 mice without tumors were treated in the same way as those in the nude mouse experiment. After the last treatment, animals were killed and underwent an autopsy study, and their organs were harvested for histopathological analysis as previously described. ${ }^{16}$ Tissue samples were taken from the liver, kidney, brain, spleen, and skin. All tissue samples were embedded in optimal cutting temperature compound (Tissue-Tek, Electron Microscopy Sciences, Washington, PA), snapfrozen in liquid nitrogen, and stored at $-80^{\circ} \mathrm{C}$ until they were sectioned at a later date. Four serial sections were cut at $4-\mu \mathrm{m}$ intervals, thaw-mounted onto glass slides, fixed with acetone for 5 minutes, and stained with $\mathrm{H} \& \mathrm{E}$ prior to histopathological examination. ${ }^{14}$

\section{Statistical Analysis and Reproducibility}

Comparisons of continuous data within groups were made using the Student t-test. ${ }^{14,15}$ All statistical tests were two-sided. In vitro experiments were repeated at least twice, and in vivo experiments were repeated at least once.

\section{Results}

\section{Purity of DTAT13 and DTAT}

To determine the size and purity of DTAT and DTAT13, we isolated both recombinant agents on a Q Sepharose anion-exchange column and performed a sodium dodecyl sulfate-polyacrylamide gel electrophoresis analysis. The analysis showed that DTAT13 was more than $90 \%$ pure and had a molecular weight of approximately $71 \mathrm{kD}$ and that the purity of DTAT exceeded $95 \%$ with a molecular weight of $58 \mathrm{kD} .{ }^{14,15}$ The protein yield for DTAT 13 was $0.7 \mathrm{mg} / \mathrm{L}$ and was $1.25 \mathrm{mg} / \mathrm{L}$ for DTAT. Control fusion proteins were tested for purity and molecular weight in a similar manner.

\section{Cytotoxicity of DTAT13 and DTAT on GBM}

To determine the cytotoxicity of DTAT13 and DTAT on GBM cell lines, a tritiated thymidine incorporation assay was performed. The GBM cell lines U373MG and U87MG were incubated with increasing concentrations of DTAT13 for 72 hours. Both glioma cell lines were inhibited in a dose- 
dependent manner by DTAT13 with an $\mathrm{IC}_{50}$ of $0.2 \mathrm{nM}$ for U87MG cells and $0.0007 \mathrm{nM}$ for U373MG cells. ${ }^{14}$ For U87MG cells, the $\mathrm{IC}_{50}$ for DTAT was $0.24 \mathrm{nM}$, which was similar to the $\mathrm{IC}_{50}$ of $0.23 \mathrm{nM}$ for DTAT13 (Fig. 2). AntiIL13 and anti-uPAR antibodies were able to block the cytotoxicity of DTAT13, indicating that the ATF molecule and the IL-13 moiety retained their binding activity. These blocking studies indicate that the antitumor effect of DTAT13 is mediated through both the IL-13 and ATF portions of the molecule. The U118MG cells responded in a dose-dependent fashion to DTAT13 in cases in which the $\mathrm{IC}_{50}$ was 0.12 nM. The control cell line Daudi, a B cell lymphoma, was not affected by DTAT13 or DTAT. Also, SKBR3 cells were not inhibited by DTAT, and Neuro2a was only inhibited at the highest concentration of $10 \mathrm{nM}$. To determine the maximum period for cytotoxicity, we incubated DTAT with U118MG cells for 24, 48, and 72 hours. Dose-response studies demonstrated that maximum cytotoxicity was reached at 48 hours and was not enhanced by longer exposure to DTAT for 72 hours. ${ }^{15}$

\section{Cytotoxicity of DTAT13 and DTAT for Endothelial Cells}

To measure the ability of DTAT to bind to HUVECs, DTAT was incubated with HUVECs in various concentrations. It was found that DTAT inhibited HUVEC in a dose-dependent manner with an $\mathrm{IC}_{50}$ of $2 \mathrm{nM} .{ }^{15}$ Control fusion proteins were not inhibitory. Also, DTAT13 inhibited HUVECs in a dose-dependent manner, demonstrating that the ATF portion of the molecule retains its killing ability selectively by binding to uPAR. To determine whether the activity of DTAT on HUVECs was mediated by the ATF, the DTAT was preincubated with polyclonal antiurokinase antibody before it was added to the HUVECs. Antiurokinase antibody blocked the inhibition by $1-$ and 10-nM concentrations of DTAT.

\section{In Vivo Antitumor Activity of DTAT and DTAT13}

To study the in vivo efficacy of DTAT, we established subcutaneous human GBM tumors in a nude mouse model using U118MG cells. On Day 30, after the tumors were established and had reached a size of $0.2 \mathrm{~cm}^{3}$, animals in groups of five received $20 \mu \mathrm{g}$ /day of DTAT injected into the tumors every other day for a total of five doses. ${ }^{15}$ Control tumors were treated with PBS or DT-IL2. By Day 48, there was a statistically significant difference $(p=0.05)$ in the size of tumors in the animals that were treated compared with those of control animals. Tumors in the DTAT-treated group progressively decreased in size, and there was only one tumor that did not completely regress by Day 65 .

To test the effectiveness of DTAT13 in vivo, we established human GBM subcutaneous flank tumors in nude mice using $6 \times 10^{6}$ U373MG and U87MG cells. The U373MG tumors were treated every other day for five doses with 10 $\mu \mathrm{g} /$ dose of DTAT13, and U87MG tumors received either DTAT or DTAT13 using the same dosage and treatment schedule. ${ }^{14}$ Treatment with DTAT13 resulted in statistically significant regression $(p<0.05)$ of U373MG tumors in treated animals after 21 to 30 days of treatment compared with tumors in control animals. All U373MG tumors treated with DTAT13 completely regressed by Day 30, and after 105 days three animals remained tumor free. When U87MG flank tumors were treated with DTAT or DTAT13,

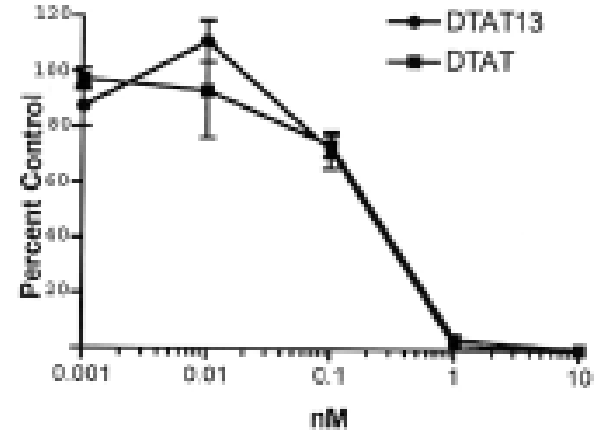

FIG. 2. Activity of DTAT and DTAT13 on a proliferating U87MG human glioblastoma cell line. Cells were cultured with fusion proteins for 72 hours, and proliferation was measured by uptake of tritiated thymidine. Data are percentages of the control response (the response of untreated cells). Data are expressed as means \pm standard deviations. Mean control value \pm standard deviation for U87MG cells was 24,734 \pm 5092 .

statistically significant tumor regression $(\mathrm{p}<0.05)$ was seen 16 to 26 days after beginning treatment compared with tumors in control animals (Fig. 3). Tumor regression was rapid, but two of five mice in the DTAT-treated group showed recurrence on Days 20 to 30 compared with the DTAT13-treated group, in which recurrence was seen in three of five animals by 40 to 50 days after beginning treatment. Two DTAT13-treated mice remained tumor free after 60 days. The DTAT13 had inhibitory effects on U373MG tumors and appeared to be more effective than DTAT on U87MG flank tumors.

\section{Systemic Toxicity of DTAT and DTAT13}

To determine the systemic toxicity of DTAT and DTAT13, the body weight of mice that received five intratumoral injections was measured. Because of the difference in the size of the two molecules, mice received $20 \mu \mathrm{g}$ of DTAT and $24 \mu \mathrm{g}$ of DTAT13 per injection. ${ }^{14}$ Animals that were treated with DTAT13 did not lose any weight compared with control animals, and mice treated with DTAT lost weight in the same comparison. Mice treated with DTAT had an average starting weight of approximately $29.8 \mathrm{~g}$, which decreased to an average of $24.2 \mathrm{~g}$ after the last injection. Even mice that received only $10-\mu \mathrm{g}$ injections of DTAT demonstrated weight loss compared with control or DTAT13-treated mice.

We also evaluated systemic toxicity in mice receiving intracranial injections of DTAT and DTAT13. Mice in groups of 10 that received $24 \mu \mathrm{g}$ of DTAT13 divided into three $8-\mu \mathrm{g}$ injections given every other day did not demonstrate weight loss compared with DTAT-treated mice in which the greatest concentration that was tolerated was $0.15 \mu \mathrm{g}$ given every other day for three doses. Overall, DTAT13 was 160fold less toxic than DTAT when given either intratumorally or intracranially. ${ }^{14}$

\section{Organ Toxicity}

Mice with U373MG flank tumors received five $10-\mu \mathrm{g}$ injections of DTAT13 and had their internal organs examined for toxicity. Tissue samples were taken from the organs, then frozen and stained with $\mathrm{H} \& \mathrm{E}$ for microscopic examination. Kidney tissue demonstrated minor neutrophil 


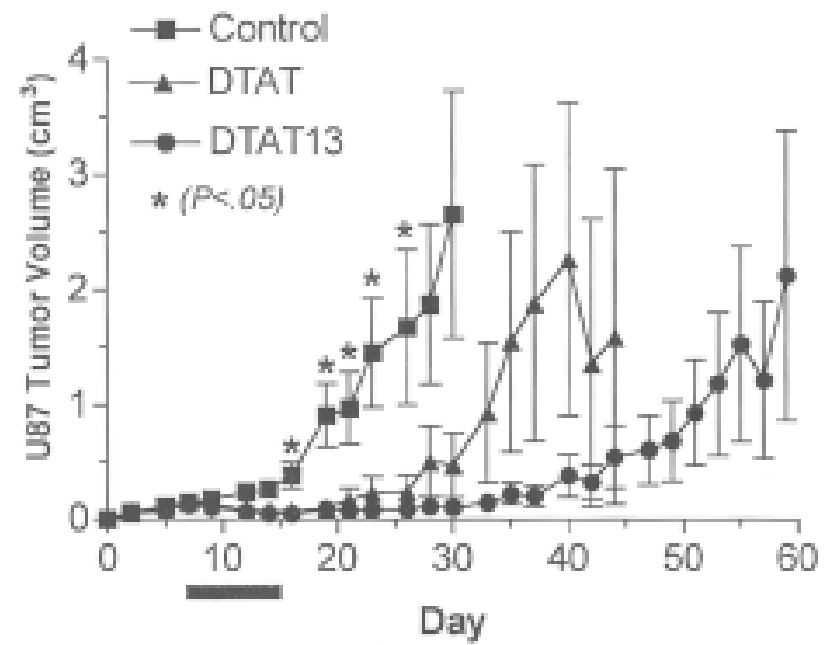

FIG. 3. Regression of U87MG glioblastoma tumors following intratumoral injection of DTAT and DTAT13. Nude mice (five per group) were injected subcutaneously with $6 \times 10^{6} \mathrm{U} 87 \mathrm{MG}$ cells on Day 0. Mice treated with DTAT or DTAT13 received five intratumoral injections every other day at $10 \mu \mathrm{g} /$ day starting on Day 6 postinjection. Treatment duration is indicated by the solid black line below the abscissa. Tumors on control mice were not injected. Tumor size was monitored every 2 to 3 days. Tumor volume $\left(\mathrm{cm}^{3}\right)$ was expressed as mean \pm one standard error unit. Tumor volume was significantly decreased $(\mathrm{p}<0.05)$ in DTAT- and DTAT13treated mice compared with control mice on Days 15 to 26. Control mice were killed on Day 30 of the experiment because of the large tumor size.

infiltration with glomeruli that appeared normal. ${ }^{14}$ Minor neutrophil infiltration was also seen in the liver tissue (Fig. 4). Brain, spleen, and skin tissues were not seen to be affected by subcutaneous tumor injection of DTAT13. Intracranial injection of DTAT13 had no effect on liver or kidney tissues.

To assess organ toxicity, C57BL/6 mice without tumors received a total of five subcutaneous doses of $20 \mu \mathrm{g}$ of DTAT given every other day. ${ }^{15}$ Tissue samples were taken from the organs, frozen and stained with $\mathrm{H} \& \mathrm{E}$, and functional assays were performed on the serum. Examination of the kidney tissue showed minor neutrophil infiltration with glomeruli that appeared healthy. The liver tissue showed minor neutrophil and mononuclear infiltration. Heart and spleen tissues were not affected, and there was minor neutrophil infiltration in the lung tissue. Functional analysis showed a statistically significant $(\mathrm{p}=0.046)$ elevation of the serum ALT level. The serum BUN level was not influenced by DTAT. The nephrotoxic control-targeted toxin $\mathrm{DT}_{390}$-anti-CD3sFv caused significant elevation of the serum BUN, and glomerular damage and tubular rhexis was noted on examination of kidney tissue.

\section{Discussion}

In this study we describe the design and synthesis of two new anti-GBM targeted toxins, DTAT and DTAT13, and compare their efficacy in vitro and in vivo. Our results show that DTAT has the advantage of targeting the UPAR expressed on both tumor cells and the neovasculature sup-

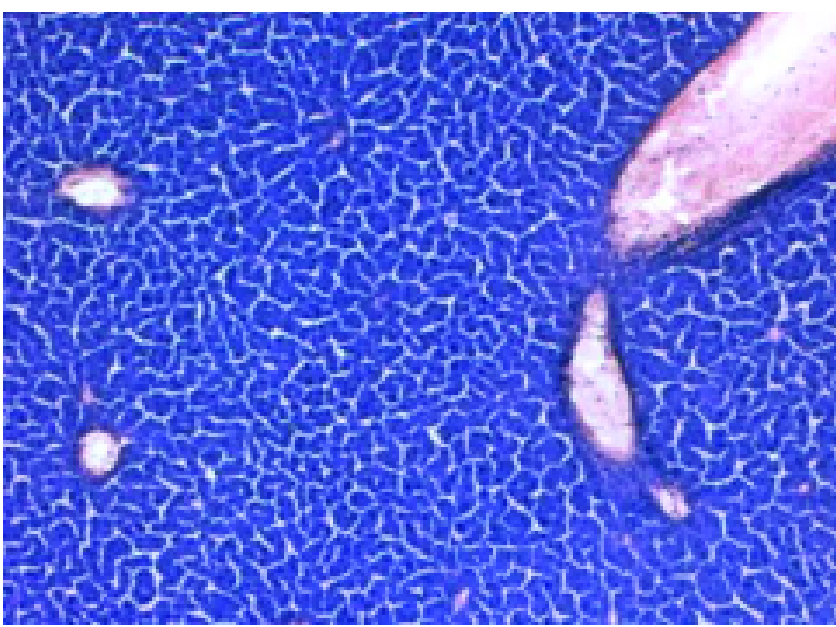

FIG. 4. Photomicrograph showing histological features of the liver from a DTAT13-treated mouse. The livers of treated mice receiving $10 \mu \mathrm{g} /$ day were examined. Tissues were removed, sectioned, and stained with $\mathrm{H} \& \mathrm{E}$. Three animals per group were examined with the same results. Original magnification $\times 100$.

plying the tumor and that DTAT13 targets UPAR, as does DTAT and the IL-13 receptors expressed on GBM cells. Both agents were shown to kill GBM cells in vitro and in vivo and HUVECs in vitro; however, neither agent was able to kill all GBM cell lines. These results confirm the heterogeneous nature of GBM and explain why no single targeted toxin is likely to be universally effective. In addition, these results also help to explain the findings demonstrated in some of the ongoing clinical trials that use targeted toxins against GBM. In Phase II trials that use a mutated DT directed against the transferrin receptor, patients who are considered responsive to the agent survived significantly longer than did those who did not respond. ${ }^{6}$

Antibodies to UPAR and IL-13 receptors were able to block the activity of DTAT13. For patients expressing both cell-surface antigens, the bispecific protein might demonstrate enhanced entry into the cytosol, which could lead to the need for administration of a smaller amount of a drug to produce a therapeutic effect. A decrease in the required dose of a targeted toxin to achieve a therapeutic effect should decrease the possibility of systemic toxicity related to these agents. Our previous work has demonstrated that the toxicity is related to DT moiety. Therefore, by administering a smaller amount of a toxin, we should be able to achieve the same therapeutic response with lower systemic toxicity.

Both DTAT and DTAT13 were effective against human GBM flank tumors in mice by targeting the tumor cells because the neovasculature that was present was mouse-derived and DTAT is not cross-reactive among species. ${ }^{10}$ Even without the antivascular effect, both DTAT and DTAT13 appear to be therapeutically effective. The catalytic component of DT and its translocation-enhancing region improved the ability of the ATF to internalize and enabled both DTAT and DTAT13 to act as potent fusion proteins.

The development of agents that target tumor vasculature is an area of intense interest to other investigators because of the added potential to deprive a growing tumor of its necessary nutrients. A VEGF-DT fusion immunotoxin has 
been assembled and shows great potential in GBM. ${ }^{9}$ The VEGF immunotoxin is important in tumor neovascularization and the development of tumor stroma, and VEGF receptors are overexpressed on the endothelial cells of tumor neovasculature. ${ }^{14}$ The immunotoxin derives its antitumor effect through vascular-mediated injury at the tumor site, which could be deleterious in the brain. Intracranial bleeding and vascular occlusion in the brain may preclude the use of VEGF receptor-based immunotoxins. Fusion proteins directed against uPAR may be more appropriate for targeting the endothelial cells in the tumor vasculature because immunohistochemical studies have shown that UPAR are primarily expressed in tissues undergoing extensive remodeling, such as the cells of primary tumor and metastasis. ${ }^{13}$ Compared with normal endothelium, uPAR may be preferentially expressed in proliferating endothelium.

Both DTAT and DTAT13 had some effect on the liver but had no effect on other organs. These systemic toxicity studies on DTAT and DTAT13 were performed in mice without tumors, suggesting that it might be possible to administer even higher doses of the fusion proteins before causing toxicity because the tumor could act as an "antigenic sink," thereby limiting the dose taken up by other nontarget organs..$^{15}$ In mice without tumors, more toxic stress is placed on the liver, which explains the elevation in ALT seen with DTAT and DTAT13. The fact that higher doses of DTAT and DTAT13 are tolerated in tumor-bearing mice compared with nontumor-bearing animals suggests that a therapeutic window exists, which further supports the rationale for developing these fusion proteins.

When injected subcutaneously or intracranially, DTAT13 was 160 -fold less toxic than DTAT. ${ }^{14}$ The larger size of the DTAT13 molecule might provide it with an additional advantage over DTAT because it is not as easily filtered through the kidney and it does not present an increased risk of hepatic toxicity..$^{14}$ Larger fusion proteins might have a reduced risk of toxicity because they might show favorable pharmacokinetic properties compared with smaller conjugates. Therapeutically, DTAT13 performed better than DTAT in preventing regrowth in tumor-bearing animals. It is our hope that this result is due to the ability of DTAT13 to target both the tumor and its vasculature. The beneficial effects of DTAT and DTAT13 in vitro and in vivo in a subcutaneous GBM tumor model have now paved the way to determine whether these same encouraging results can be achieved in an intracranial tumor model.

\section{References}

1. Appella E, Robinson EA, Ullrich SJ, et al: The receptor-binding sequence of urokinase. A biological function for the growth factor module of proteases. J Biol Chem 262:4437-4440, 1987

2. Hall WA: Targeted toxin therapy for malignant astrocytoma. Neurosurgery 46:544-552, 2000
3. Hall WA, Rustamzadeh E, Asher AL: Convection-enhanced delivery in clinical trials. Neurosurg Focus 14(2):E2, 2003

4. Jain RK: Delivery of novel therapeutic agents in tumors: physiological barriers and strategies. J Natl Cancer Inst 81:570-576, 1989

5. Kroon ME, Koolwijk P, van der Vecht B, et al: Urokinase receptor expression on human microvascular endothelial cells is increased by hypoxia: implications for capillary-like tube formation in a fibrin matrix. Blood 96:2775-2783, 2000

6. Laske DW, Youle RJ, Oldfield EH: Tumor regression with regional distribution of targeted toxin TF-CRM107 in patients with malignant brain tumors. Nat Med 3:1362-1368, 1997

7. Li C, Hall WA, Jin N, et al: Targeting glioblastoma multiforme with an IL-13/diphtheria toxin fusion protein in vitro and in vivo in nude mice. Protein Eng 15:419-427, 2002

8. Mori T, Abe T, Wakabayashi Y, et al: Up-regulation of urokinase-type plasminogen activator and its receptor correlates with enhanced invasion activity of human glioma cells mediated by transforming growth factor- $\alpha$ of basic fibroblast growth factor. J Neurooncol 46:115-123, 2000

9. Olson TA, Mohanraj D, Roy S, et al: Targeting the tumor vasculature: inhibition of tumor growth by a vascular endothelial growth factor-toxin conjugate. Int J Cancer 73:865-870, 1997

10. Quax PHA, Grimbergen JM, Lansink M, et al: Binding of human urokinase-type plasminogen activator to its receptor. Residues involved in species specificity and binding. Arterioscler Thromb Vasc Biol 18:693-701, 1998

11. Ramakrishnan S, Olson TA, Bautch VL, et al: Vascular endothelial growth factor-toxin conjugate specifically inhibits KDR/ flk-1-positive endothelial cell proliferation in vitro and angiogenesis in vivo. Cancer Res 56:1324-1330, 1996

12. Rustamzadeh E, Low WC, Vallera DA, et al: Immunotoxin therapy for CNS tumor. J Neurooncol 64:101-116, 2003

13. Solberg H, Ploug M, Hoyer-Hansen G, et al: The murine receptor for urokinase-type plasminogen activator is primarily expressed in tissues actively undergoing remodeling. J Histochem Cytochem 49:237-246, 2001

14. Todhunter DA, Hall WA, Rustamzadeh E, et al: A bispecific immunotoxin (DTAT13) targeting human IL-13 receptor (IL-13R) and urokinase-type plasminogen activator receptor (u-PAR) in a mouse xenograft model. Protein Eng Des Sel 17:157-164, 2004

15. Vallera DA, Li C, Jin N, et al: Targeting urokinase-type plasminogen activator receptor on human glioblastoma tumors with diphtheria toxin fusion protein DTAT. J Natl Cancer Inst 94: 597-606, 2002

16. Vallera DA, Panoskaltsis-Mortari A, Blazar BR, et al: Renal dysfunction accounts for the dose limiting toxicity of $\mathrm{DT}_{390}$ anti$\mathrm{CD} 3 \mathrm{sFv}$, a potential new recombinant anti-GVHD immunotoxin. Protein Eng 10:1071-1076, 1997

17. Vallera DA, Panoskaltsis-Mortari A, Jost C, et al: Anti-graftversus-host disease effect of $\mathrm{DT}_{390}$-anti-CD3sFv, a single-chain Fv fusion immunotoxin specifically targeting the CD $3 \epsilon$ moiety of the T-cell receptor. Blood 88:2342-2353, 1996

Manuscript received January 30, 2006.

Accepted in final form March 16, 2006.

Address reprint requests to: Walter A. Hall, M.D., MMC \#96, 420

Delaware Street SE, Minneapolis, Minnesota 55455. email: hallx003 @umn.edu. 